\title{
MASS TRANSPORT PROBLEMS OBTAINED AS LIMITS OF $p$-LAPLACIAN TYPE PROBLEMS WITH SPATIAL DEPENDENCE
}

\author{
J. M. MAZÓN, J. D. ROSSI, AND J. TOLEDO
}

\begin{abstract}
We consider the following problem: given a bounded convex domain $\Omega \subset \mathbb{R}^{N}$ we consider the limit as $p \rightarrow \infty$ of solutions to $-\operatorname{div}\left(b_{p}^{-p}|D u|^{p-2} D u\right)=f_{+}-f_{-}$, in $\Omega, b_{p}^{-p}|D u|^{p-2} \frac{\partial u}{\partial \eta}=0$, on $\partial \Omega$. Under appropriate assumptions on the coefficients $b_{p}$, that in particular verify that $\lim _{p \rightarrow \infty} b_{p}=b$ uniformly in $\bar{\Omega}$, we prove that there is a uniform limit of $u_{p_{j}}$ (along a sequence $p_{j} \rightarrow \infty$ ) and that this limit is a Kantorovich potential for the optimal mass transport problem of $f_{+}$to $f_{-}$ with cost $c(x, y)$ given by the formula $c(x, y)=\inf _{\sigma(0)=x, \sigma(1)=y} \int_{\sigma} b d s$.
\end{abstract}

\section{INTRODUCTION}

Since the work by Evans and Gangbo on optimal mass transportation, [5], the idea of taking limits as $p \rightarrow \infty$ in $p$-Laplacian type problems to find solutions to optimal mass transport problems was recently used by the authors of this paper, see $[6,7,8,9]$. Our main goal in the present paper is to see what are the optimal transport problems that can be approximated when one considers a spatially dependent coefficient in the $p$-Laplacian approximations.

Associated with a positive and continuous function $b_{p}$ in $\bar{\Omega}$ as diffusion coefficient let us consider the following $p$-Laplacian type problem:

$$
\begin{cases}-\operatorname{div}\left(b_{p}^{-p}|D u|^{p-2} D u\right)=f, & \text { in } \Omega, \\ b_{p}^{-p}|D u|^{p-2} \frac{\partial u}{\partial \eta}=0, & \text { on } \partial \Omega .\end{cases}
$$

Here $\Omega$ is a bounded and convex domain in $\mathbb{R}^{N}$ and $f=f_{+}-f_{-} \in L^{\infty}(\Omega)$ has zero mean in $\Omega, \int_{\Omega} f=0$ (otherwise this problem does not have solutions). Concerning the coefficient $b_{p}$ we also assume that there exists a continuous positive function $b$ in $\bar{\Omega}$ such that

$$
b_{p}^{p} \leq \frac{B^{p}}{c_{1}}, \quad b^{p} \leq c_{2} b_{p}^{p}
$$

Date: September 30, 2013.

Key words and phrases. Mass transport, Monge-Kantorovich problems, p-Laplacian equation.

2000 Mathematics Subject Classification. 49J20,49J45,45G10. 
for any $p$ and for fixed constants $B, c_{1}, c_{2}>0$, and moreover, it holds that

$$
\lim _{p \rightarrow \infty} b_{p}=b
$$

uniformly in $\bar{\Omega}$. The simplest example of positive $b_{p}$ that verifies (1.3) is to consider $b_{p}$ independent of $p, b_{p}(x)=b(x)$, for a fixed positive continuous function on $\bar{\Omega}$.

Existence and uniqueness (up to an additive constant) for this problem of a continuous weak solution in the Sobolev space $W^{1, p}(\Omega)$ can be easily obtained from variational arguments. It turns out that this weak solution is also a viscosity solution, see [10].

Limits as $p \rightarrow \infty$ of similar type problems are related to optimal mass transport problems for the Euclidean distance. In fact, this relation was the key to the first complete proof of the existence of an optimal transport map for the classical Monge problem (here the transport cost of one unit of mass between $x$ and $y$ is the Euclidean distance $|x-y|$ ) given by Evans and Gangbo in [5]. Note that the usual Euclidean distance is not a strictly convex cost. This makes this optimal mass transport different from the strictly convex cost case in which there is existence of a convex function (solution to a Monge Ampere type problem) whose gradient provides an optimal transport map, see [11]. For notation and general results on Mass Transport Theory we refer to $[1,2,4,5,11]$ and [12].

In our case, we can pass to the limit in (1.1) and obtain that, for a sequence $p_{j} \rightarrow \infty, u_{p_{j}} \rightrightarrows u_{\infty}$ uniformly in $\bar{\Omega}$. It turns out that this limit $u_{\infty}$ is a Kantorovich potential for the optimal transport problem that we describe below.

An optimal mass transport problem with a non-standard cost. Assume that we have some production in a domain $\Omega$ encoded in $f_{+}$and some consumption encoded in $f_{-}$. To transport one unit of material from $x$ to $y$ we pay as transport $\operatorname{cost} c(x, y)$ (that may take into account that the cost is not translation invariant in this transport operation) that in our case is given in terms of $b$ by the formula

$$
c(x, y)=\inf _{\sigma \in \Gamma(x, y)} \int_{\sigma} b d s,
$$

where $\Gamma(x, y):=\left\{\sigma \in C^{1}([0,1], \Omega): \sigma(0)=x, \sigma(1)=y\right\}$.

Our main goal is to move the whole production and satisfy the whole demand minimizing the total cost of the operation.

In its relaxed version (Monge-Kantorovich problem), this optimal transport problem reads as follows: Let $\Pi\left(f_{+}, f_{-}\right)$be the set of transport plans between $f_{+}$and $f_{-}$, that is, the set of nonnegative Radon measures $\mu$ in $\Omega \times \Omega$ such that $\operatorname{proj}_{x}(\mu)=f_{+} d x$ and $\operatorname{proj}_{y}(\mu)=f_{-} d x$; the aim is to find a 
measure $\mu^{*} \in \Pi\left(f_{+}, f_{-}\right)$which minimizes the cost functional

$$
\mathcal{K}_{c}(\mu):=\int_{\Omega \times \Omega} c(x, y) d \mu(x, y)
$$

in the set $\Pi\left(f_{+}, f_{-}\right)$.

We have the following result:

Theorem 1.1. Let $u_{p}$ be the unique solution to (1.1) that verifies $\int_{\Omega} u_{p}=0$ and assume (1.2) and (1.3). Then, there is a sequence $p_{j} \rightarrow \infty$ such that the uniform limit of the solutions $u_{p_{j}}, u_{\infty}$, is a Kantorovich potential for the optimal transport problem of $f_{+}$to $f_{-}$with the cost given by $c(x, y)$ in (1.4), that is,

$$
\min \left\{\mathcal{K}_{c}(\mu): \mu \in \Pi\left(f_{+}, f_{-}\right)\right\}=\sup \left\{\int_{\Omega} v f: v \in K_{c}(\Omega)\right\}=\int_{\Omega} u_{\infty} f,
$$

where

$$
K_{c}(\Omega):=\{v: \Omega \rightarrow \mathbb{R}:|v(x)-v(y)| \leq c(x, y), \quad \forall x, y \in \Omega\} .
$$

Note that we can approximate the total transport cost since we have that

$$
\lim _{p_{j} \rightarrow \infty} \int_{\Omega} u_{p_{j}} f=\int_{\Omega} u_{\infty} f
$$

Let us end the introduction with a brief description of the main techniques used in the proofs. Concerning approximations using $p$-Laplacian type operators, we quote [3], from where the main idea to show the key bounds for the $L^{p}$-norm of the gradient is taken. Once we have a uniform in $p$ bound for the $L^{p}$-norm of the gradients we can extract a subsequence that converge uniformly and show that this limit is a maximizer of $\int_{\Omega} v f d x$ in $K_{1}(\Omega)$. From this the proof follows using the general duality argument that can be found, for example, in [11].

When $b_{p}$ is of the form $b_{p}=B e^{-\eta}, \eta>0$, for the study of the limit equation (in the viscosity sense) when $p \rightarrow \infty$ we refer to [10]. Here we focus our attention on the mass transport problem obtained in this limit procedure rather than in the equation that is verified by the limit.

The paper is organized as follows: In Section 2 we prove that there is a sequence of solutions to (1.1) that converges uniformly; in Section 3 we prove that the uniform limit is a solution (Kantorovich potential) to the optimal mass transport problem.

\section{A $p$-LAPLACIAN LIMIT}

Recall that we are considering problem (1.1). First, we show existence and uniqueness to it. The proof is standard but we include the details for the sake of completeness. 
Lemma 2.1. Let $p>N$ be fixed, then there it exists a unique continuous solution to the variational problem

$$
\min _{S_{p}}\left\{\int_{\Omega} \frac{1}{b_{p}^{p}} \frac{|D u|^{p}}{p}-\int_{\Omega} u f\right\}
$$

where

$$
S_{p}=\left\{u \in W^{1, p}(\Omega): \int_{\Omega} u=0\right\} .
$$

This minimum is a weak solution of problem (1.1), that is, it verifies

$$
\int_{\Omega} \frac{1}{b_{p}^{p}}|D u|^{p-2} D u D \phi=\int_{\Omega} f \phi, \quad \forall \phi \in C^{\infty}(\Omega) .
$$

Proof. By our assumptions we have that $b_{p}^{-p}$ is bounded from below and above, $0<c_{1} B^{-p} \leq b_{p}^{-p} \leq c_{2, p}<\infty$ (note that even $c_{1}$ can depend on $p$ here since $p$ is fixed along this proof). Hence, we obtain that for every $u$ $\in W^{1, p}(\Omega)$ there holds

$$
c_{1} B^{-p} \int_{\Omega} \frac{|D u|^{p}}{p} \leq \int_{\Omega} \frac{1}{b_{p}^{p}} \frac{|D u|^{p}}{p} \leq c_{2, p} \int_{\Omega} \frac{|D u|^{p}}{p}
$$

and then the functional

$$
\Theta(u)=\int_{\Omega} \frac{1}{b_{p}^{p}} \frac{|D u|^{p}}{p}-\int_{\Omega} u f,
$$

is well defined in the set $S_{p}$ which is convex, weakly closed and non empty. On the other hand, $\Theta$ is coercive, bounded below and lower semicontinuous in $S_{p}$, for this reason there is a minimizing sequence $u_{n} \in S_{p} \subset W^{1, p}(\Omega)$, such that $u_{n} \rightarrow u \in S_{p}$, and

$$
\inf _{S} \Theta=\liminf _{n \rightarrow+\infty} \Theta\left(u_{n}\right) \geq \Theta(u) .
$$

Hence the minimum of $\Theta$ in $S_{p}$ is attained. From the strict convexity of $\Theta$ in $S_{p}$ we obtain that $u_{p}$ is the unique minimum of $\Theta$ in $S_{p}$. Finally, $u_{p}$, the unique minimizer, is a weak solution of (1.1). The fact that $u_{p}$ is continuous follows from the fact that $W^{1, p}(\Omega) \hookrightarrow C(\bar{\Omega})$ since $p>N$.

Remark 2.1. Note that we have imposed that $\int_{\Omega} u=0$ just to obtain uniqueness of the solution. As usually happens for homogeneous Neumann problems there are infinitely many solutions to (1.1) but any two of them differ by an additive constant.

Remark 2.2. Following the ideas in [10] it can be proved that a continuous weak solution to (1.1) is a viscosity solution to the same equation.

Our next step is to prove that we can extract a sequence of solutions to (1.1), $\left\{u_{p_{j}}\right\}_{j}$, with $p_{j} \rightarrow \infty$, that converges uniformly as $j \rightarrow \infty$. 
Lemma 2.2. Let $u_{p}$ be solution to (1.1), $p>N$. There exists a sequence $p_{j} \rightarrow \infty$ such that

$$
u_{p_{j}} \rightrightarrows u_{\infty}
$$

uniformly in $\bar{\Omega}$. Moreover, the limit $u_{\infty}$ is Lipschitz continuous.

Proof. Along this proof we will denote by $C$ a constant independent of $p$ that may change from one line to another.

Our first aim is to prove that the $L^{p}$-norm of the gradient of $u_{p}$ is bounded independently of $p$. We already proved in the previous Lemma 2.1 that $u_{p}$ is a minimizer of $\Theta$ in $S_{p}$. Let $v$ a fixed Lipschitz function with $|D v| \leq b$ a.e. in $\Omega$ and $\int_{\Omega} v=0$, then we have that $v \in S_{p}$ and hence

$$
\begin{aligned}
\int_{\Omega} \frac{1}{b_{p}^{p}} \frac{\left|D u_{p}\right|^{p}}{p}-\int_{\Omega} f u_{p} & \leq \int_{\Omega} \frac{1}{b_{p}^{p}} \frac{|D v|^{p}}{p}-\int_{\Omega} f v \\
& \leq \int_{\Omega} \frac{b^{p}}{p b_{p}^{p}}-\int_{\Omega} f v .
\end{aligned}
$$

Now, from (1.2) we have

$$
\int_{\Omega} \frac{1}{b_{p}^{p}} \frac{\left|D u_{p}\right|^{p}}{p} \leq \frac{c_{2}}{p}|\Omega|-\int_{\Omega} f v+\int_{\Omega} f u_{p} .
$$

Thanks to the fact that $\int_{\Omega} u_{p}=0$ (and the fact that the constant in the inequality $\left\|u_{p}\right\|_{L^{p}(\Omega)} \leq C\left\|D u_{p}\right\|_{L^{p}(\Omega)}$ can be chosen independent of $p$, see [8]) we get,

$$
\int_{\Omega} f u_{p} \leq C\left\|u_{p}\right\|_{L^{p}(\Omega)} \leq C\left\|D u_{p}\right\|_{L^{p}(\Omega)}
$$

and then we get

$$
\int_{\Omega} \frac{1}{b_{p}^{p}} \frac{\left|D u_{p}\right|^{p}}{p} \leq C+C\left\|D u_{p}\right\|_{L^{p}(\Omega)} .
$$

Now we use that $b_{p}^{-p} \geq c_{1} B^{-p}$ to obtain

$$
\int_{\Omega}\left|D u_{p} / B\right|^{p} \leq p C+p C\left\|D u_{p} / B\right\|_{L^{p}(\Omega)} .
$$

From this inequality we can obtain that there exists $C$, independent of $p$, such that

$$
\left(\int_{\Omega}\left|D u_{p}\right|^{p}\right)^{\frac{1}{p}} \leq C .
$$

Now, using this uniform bound, we prove uniform convergence of a sequence $u_{p_{j}}$. In fact, we take $m$ such that $N<m \leq p$ and obtain the 
following bound

$$
\begin{aligned}
\left\|D u_{p}\right\|_{L^{m}(\Omega)} & =\left(\int_{\Omega}\left|D u_{p}\right|^{m} \cdot 1\right)^{\frac{1}{m}} \\
& \leq\left[\left(\int_{\Omega}\left|D u_{p}\right|^{p}\right)^{\frac{m}{p}}\left(\int_{\Omega} 1\right)^{\frac{p-m}{p}}\right]^{\frac{1}{m}} \\
& \leq C_{1}|\Omega|^{\frac{p-m}{p m}} \leq C_{2},
\end{aligned}
$$

the constant $C_{2}$ being independent of $p$. We have proved that $\left\{u_{p}\right\}_{p>N}$ is bounded in $W^{1, m}(\Omega)$, and we know that $\int_{\Omega} u_{p}=0$, so we can obtain a weakly convergent sequence $u_{p_{j}} \rightarrow u_{\infty} \in W^{1, m}(\Omega)$ with $p_{j} \rightarrow+\infty$. Since $W^{1, p}(\Omega) \hookrightarrow C^{0, \alpha}(\bar{\Omega})$ and $u_{p_{j}} \rightarrow u_{\infty} \in W^{1, p}(\Omega)$, we obtain $u_{p_{j}} \rightarrow u_{\infty}$ in $C^{0, \alpha}(\Omega)$, and in particular $u_{p_{j}} \rightrightarrows u_{\infty}$ uniformly in $\bar{\Omega}$. As $u_{p_{j}} \in C(\bar{\Omega})$, then $u_{\infty} \in C(\bar{\Omega})$. Using a diagonal procedure we conclude the existence of sequence $u_{p_{j}}$ that is weakly convergent in $W^{1, m}(\Omega)$ for every $m$.

Finally, let us show that the limit function $u_{\infty}$ is Lipschitz. In fact, we proved that,

$$
\left(\int_{\Omega}\left|D u_{\infty}\right|^{m}\right)^{\frac{1}{m}} \leq \liminf _{p_{j} \rightarrow+\infty}\left(\int_{\Omega}\left|D u_{p_{j}}\right|^{m}\right)^{\frac{1}{m}} \leq C_{1}|\Omega|^{\frac{1}{m}} \leq C_{2} .
$$

Now, we take $m \rightarrow \infty$ to obtain $\left\|D u_{\infty}\right\|_{L^{\infty}(\Omega)} \leq C_{2}$. So, we have proved $u_{\infty} \in W^{1, \infty}(\Omega)$, that is, $u_{\infty}$ is a Lipschitz function.

\section{MASs transport interpretation of the Limit}

The goal of this section is to show that $u_{\infty}$ is a Kantorovich potential for the mass transport problem of $f_{+}$to $f_{-}$with the cost $c(x, y)$ given by

$$
c(x, y)=\inf _{\sigma \in \Gamma(x, y)} \int_{\sigma} b d s
$$

that is,

$$
c(x, y)=\inf _{\sigma \in \Gamma(x, y)} \int_{0}^{1} L\left(\sigma(t), \sigma^{\prime}(t), t\right) d t,
$$

with $L$ the Lagrangian given by $L(z, \xi)=b(z)|\xi|$.

The key idea to identify the cost is as follows: if we have a Lipschitz continuous function such that

$$
|D u(x)| \leq b(x) \quad \text { a.e. in } \Omega,
$$

then, choosing a path $\sigma$ with $\sigma(0)=x, \sigma(1)=y$, and

$$
c(x, y) \geq \int_{\sigma} b d s-\varepsilon
$$


we have

$$
\begin{aligned}
|u(x)-u(y)| & =\left|\int_{0}^{l}\left\langle D u(\sigma(t)), \sigma^{\prime}(t)\right\rangle d t\right| \\
& \leq \int_{0}^{l} b(\sigma(t))\left|\sigma^{\prime}(t)\right| d t \\
& \leq c(x, y)+\varepsilon .
\end{aligned}
$$

Hence, we conclude that

$$
|u(x)-u(y)| \leq c(x, y)
$$

Conversely, if we have

$$
|u(x)-u(y)| \leq c(x, y)
$$

then

$$
|D u(x)| \leq b(x) \quad \text { a.e. in } \Omega .
$$

In fact, for $\xi \in \mathbb{R}^{N}$ and $h \in \mathbb{R}$ with $|h|$ small enough, if we just consider the path $\sigma:[0,1] \rightarrow \Omega$ given by

$$
\sigma(t)=x+t\left(h b^{-1}(x) \xi\right)
$$

we have

$$
\begin{aligned}
& \left|\left\langle b^{-1}(x) D u(x), \xi\right\rangle\right|=\left|\left\langle D u(x), b^{-1}(x) \xi\right\rangle\right| \leq \liminf _{h \rightarrow 0} \frac{c\left(x, x+h b^{-1}(x) \xi\right)}{|h|} \\
& \quad \leq \liminf _{h \rightarrow 0} \frac{1}{|h|} \int_{\sigma} b d s=\liminf _{h \rightarrow 0} \int_{0}^{1} b\left(x+t h b^{-1}(x) \xi\right) b^{-1}(x)|\xi| d t=|\xi|,
\end{aligned}
$$

from where we get (3.1).

Therefore, if $c$ and $b$ are related by

$$
c(x, y)=\inf _{\sigma \in \Gamma(x, y)} \int_{\sigma} b d s,
$$

then the set of functions

$$
K_{c}(\Omega):=\left\{u: \Omega \mapsto \mathbb{R}:|u(x)-u(y)| \leq c(x, y) \text { and } \int_{\Omega} u=0\right\}
$$

coincides with the set

$$
\tilde{K}_{b}(\Omega):=\left\{u: \Omega \mapsto \mathbb{R}:|D u(x)| \leq b(x) \text { and } \int_{\Omega} u=0\right\} .
$$

Hence, we have that

$$
\sup \left\{\int_{\Omega} v f: v \in K_{c}(\Omega)\right\}=\sup \left\{\int_{\Omega} v f: v \in \tilde{K}_{b}(\Omega)\right\} .
$$


Lemma 3.1. Any limit of a sequence $u_{p_{j}}, u_{\infty}$, is a Kantorovich potential for the optimal transport problem of $f_{+}$to $f_{-}$with the cost given by

$$
c(x, y)=\inf _{\sigma \in \Gamma(x, y)} \int_{\sigma} b d s,
$$

that is, it holds that

$$
\min \left\{\mathcal{K}_{c}(\mu): \mu \in \Pi\left(f_{+}, f_{-}\right)\right\}=\sup \left\{\int_{\Omega} v f: v \in K_{c}(\Omega)\right\}=\int_{\Omega} u_{\infty} f .
$$

Proof. The equality

$$
\min \left\{\mathcal{K}_{c}(\mu): \mu \in \Pi\left(f_{+}, f_{-}\right)\right\}=\sup \left\{\int_{\Omega} v f: v \in K_{c}(\Omega)\right\}
$$

follows by well known duality arguments, using that $c$ is a distance, see [11]. Therefore, due to (3.2), we just need to show that

$$
\sup \left\{\int_{\Omega} v f: v \in \tilde{K}_{b}(\Omega)\right\}=\int_{\Omega} u_{\infty} f .
$$

From the proof of Lemma 2.2 we have that, for every $v$ a Lipschitz function with $|D v| \leq b$ a.e. in $\Omega$ and $\int_{\Omega} v=0$, it holds

$$
\begin{aligned}
-\int_{\Omega} f u_{p} \leq \int_{\Omega} \frac{1}{b_{p}^{p}} \frac{\left|D u_{p}\right|^{p}}{p}-\int_{\Omega} f u_{p} & \leq \int_{\Omega} \frac{1}{b_{p}^{p}} \frac{|D v|^{p}}{p}-\int_{\Omega} f v \\
& \leq \int_{\Omega} \frac{b^{p}}{p b_{p}^{p}}-\int_{\Omega} f v \\
& \leq \frac{c_{2}}{p}|\Omega|-\int_{\Omega} f v
\end{aligned}
$$

Taking limits as $p \rightarrow \infty$ we obtain

$$
\int_{\Omega} f u_{\infty} \geq \sup \left\{\int_{\Omega} v f: v \in \tilde{K}_{b}(\Omega)\right\} .
$$

Then we just need to show that $u_{\infty} \in \tilde{K}_{b}(\Omega)$. From the uniform convergence of $u_{p_{j}}$ to $u_{\infty}$ we immediately conclude that $\int_{\Omega} u_{\infty}=0$. Now, using again the computations of the proof of Lemma 2.2, we get

$$
\int_{\Omega} \frac{1}{b_{p}^{p}} \frac{\left|D u_{p}\right|^{p}}{p} \leq C
$$

with $C$ independent of $p$. Hence,

$$
\left(\int_{\Omega}\left|\frac{D u_{p}}{b_{p}}\right|^{p}\right)^{1 / p} \leq(p C)^{1 / p}
$$

Since

$$
\frac{D u_{p}}{b_{p}} \rightarrow \frac{D u_{\infty}}{b}
$$


arguing as in the final part of the proof of Lemma 2.2 and using that $(p C)^{1 / p} \rightarrow 1$ as $p \rightarrow \infty$ we get

$$
\left\|b^{-1} D u_{\infty}\right\|_{L^{\infty}(\Omega)} \leq 1,
$$

that is,

$$
\left|D u_{\infty}(x)\right| \leq b(x)
$$

a.e. in $\Omega$ and we conclude that $u_{\infty} \in \tilde{K}_{b}(\Omega)$.

Remark 3.1. In one space dimension, that is $\Omega=(a, b) \subset \mathbb{R}$, it is easy to see that

$$
\begin{gathered}
\min \left\{\mathcal{K}_{c}(\mu): \mu \in \Pi\left(f_{+}, f_{-}\right)\right\}=\sup \left\{\int_{\Omega} v f: v \in K_{c}(\Omega)\right\}=\int_{\Omega} u_{\infty} f \\
=\sup \left\{\int_{\Omega} v f: v \in K_{\tilde{c}}(\Omega)\right\}
\end{gathered}
$$

where

$$
\tilde{c}(x, y):=\int_{0}^{1} b((1-t) x+t y) d t|x-y|=\int_{0}^{|x-y|} b\left(x+t \frac{y-x}{|x-y|}\right) d t
$$

being the last term 0 when $x=y$. Nevertheless, in general, for dimension greater than one, this total cost is strictly less than

$$
\min \left\{\mathcal{K}_{\tilde{c}}(\mu): \mu \in \Pi\left(f_{+}, f_{-}\right)\right\} .
$$

In one dimension, both total costs coincide, indeed, if we set $d(r)=\int_{0}^{r} b(s) d s$, then

$$
c(x, y)=\tilde{c}(x, y)=|d(x)-d(y)| .
$$

In larger dimensions this is not true in general since with the cost $\tilde{c}$ we are using straight lines to go from $x$ to $y$ and we can have functions $b$ for which a straight line is not the optimal one when computing the cost $c(x, y)$ given by (1.4).

Acknowledgments: J.M.M. and J.T. have been partially supported by the Spanish MEC and FEDER, project MTM2012-31103. J.D.R. has been partially supported by MEC MTM2010-18128 and MTM2011-27998 (Spain).

\section{REFERENCES}

[1] L. Ambrosio. Lecture notes on optimal transport problems. Mathematical aspects of evolving interfaces (Funchal, 2000), 1-52, Lecture Notes in Math., 1812, Springer, Berlin, 2003.

[2] L. Ambrosio and A. Pratelli. Existence and stability results in the $L^{1}$ theory of optimal transportation. Optimal transportation and applications (Martina Franca, 2001), 123-160, Lecture Notes in Math., 1813, Springer, Berlin, 2003.

[3] T. Bhattacharya, E. Di Benedetto and J. Manfredi. Limits as $p \rightarrow \infty$ of $\Delta_{p} u_{p}=f$ and related extremal problems. Rend. Sem. Mat. Univ. Politec. Torino, (1991), 15-68. 
[4] L. C. Evans. Partial differential equations and Monge-Kantorovich mass transfer. Current developments in mathematics, 1997 (Cambridge, MA), 65-126, Int. Press, Boston, MA, 1999.

[5] L. C. Evans and W. Gangbo, Differential equations methods for the MongeKantorovich mass transfer problem. Mem. Amer. Math. Soc., 137 (1999), no. 653.

[6] N. Igbida, J. M. Mazón, J. D. Rossi and J. Toledo. A Monge-Kantorovich mass transport problem for a discrete distance. J. Funct. Anal. 260, (2011), 3494-3534.

[7] J. M. Mazón, J. D. Rossi and J. Toledo. An optimal transportation problem with a cost given by the Euclidean distance plus import/export taxes on the boundary. To appear in Revista Matemática Iberoamericana.

[8] J. M. Mazón, J. D. Rossi and J. Toledo. An optimal matching problem for the Euclidean distance. Preprint.

[9] J. M. Mazón, J. D. Rossi and J. Toledo. Mass transport problems for the Euclidean distance obtained as limits of $p$-Laplacian type problems with obstacles. Preprint.

[10] R. López-Soriano, J. C. Navarro-Climent and J. D. Rossi. The infinity Laplacian with a transport term. J. Math. Anal. Appl. 398, (2013), 752-765.

[11] C. Villani. Topics in Optimal Transportation. Graduate Studies in Mathematics. Vol. 58, 2003.

[12] C. Villani. Optimal Transport, Old and New, Grundlehren des Mathematischen Wissenschaften (Fundamental Principles of Mathematical Sciences), Vol. 338, SpringerVerlag, Berlin-New York, 2009.

J. M. Mazón: Departament d'Anàlisi Matemàtica, Universitat de València, VALENCIA, SPAIN. mazon@uv.es

J. D. Rossi: Departamento de Análisis Matemático, Universidad de AliCante, Ap. Correos 99, 03080, Alicante, Spain and Depto. de Matemática, FCEyn UBA, Ciudad Universitaria, Pab 1 (1428), Buenos Aires, Argentina. julio.rossi@ua.es

J. Toledo: Departament D'AnÀlisi Matemàtica, Universitat de València, VAlEnCia, Spain. toledojj@uv.es 